\title{
Reduction in Al Acceptor Density by Electron Irradiation in Al-Doped 4H-SiC
}

\author{
Hideharu Matsuura ${ }^{1}$, Koichi Aso ${ }^{1}$, Sou Kagamihara ${ }^{1}$, Hirofumi Iwata ${ }^{1}$, \\ Takuya Ishida $^{1}$ and Kazuhiro Nishikawa ${ }^{2}$ \\ ${ }^{1}$ Osaka Electro-Communication University, 18-8 Hatsu-cho, Neyagawa, Osaka 572-8530, Japan \\ ${ }^{2}$ Sansha Electric Mfg. Co., Ltd., 3-1-56 Nishi-Awaji, Higashi-Yodogawa, Osaka 533-0031, Japan
}

Keywords: Electron irradiation, Hall-effect measurement, Reduction in acceptors, Temperature dependence of hole concentration, Free carrier concentration spectroscopy

\begin{abstract}
The influence of electron irradiation on the hole concentration in Al-doped 4H-SiC epilayers is investigated with free carrier concentration spectroscopy (FCCS) using the temperature dependent hole concentration $p(T)$. By $4.6-\mathrm{MeV}$ electron irradiation, $p(T)$ is reduced over the whole temperature range. Using FCCS, the densities and energy levels of acceptors or hole traps are determined. In the unirradiated and irradiated samples, $\sim 200 \mathrm{meV}$ and $\sim 370 \mathrm{meV}$ acceptor levels or hole-trap levels are detected. By irradiation, only the density of $\mathrm{Al}$ acceptors whose energy level is $\sim 200 \mathrm{meV}$ is reduced from $6.2 \times 10^{15} \mathrm{~cm}^{-3}$ to $8.2 \times 10^{14} \mathrm{~cm}^{-3}$. This indicates that the main reduction in $p(T)$ by the electron irradiation resulted from the decrease of the $\mathrm{Al}$ acceptor density, not from the creation of defects.
\end{abstract}

\section{Introduction}

$\mathrm{SiC}$ is a wide bandgap semiconductor with potential for use in high power and high frequency devices capable of operating at elevated temperatures. Also for electrons with energies greater than $0.5 \mathrm{MeV}$, the damage constant for lifetime degradation in $\mathrm{SiC}$ has been reported to be lower than that in GaAs by more than three orders of magnitude [1].

In high power Si devices [2], electron irradiation is an excellent tool for the controlled generation of intrinsic defects in Si. On the other hand, it degrades the conversion efficiency of Si solar cells used in space [3-5]. Therefore, this electron-radiation damage in Si has been investigated by many researchers. On the other hand, the understanding of radiation damage in $\mathrm{SiC}$ is far from complete.

Using electron spin resonance (ESR) spectroscopy, the following vacancy-related defects induced by electron irradiation were reported [6-8]; a Si Frenkel pair, a $\mathrm{C}$ vacancy, a divacancy, and an antisite-vacancy pair. In electron-irradiated B-doped $6 \mathrm{H}-\mathrm{SiC}$, a complex of $\mathrm{B}$ and a vacancy, which behaves as a deep acceptor [9], was detected [10]. It was reported that electrons with energies between 1 and $3 \mathrm{MeV}$ were sufficient to displace $\mathrm{Si}$ atoms in $6 \mathrm{H}-\mathrm{SiC}$ with one electron [11].

Although the radiation damage has been studied mainly with ESR and a deep level transient spectroscopy, the densities and energy levels of hole traps created by irradiation can also be determined accurately from the temperature dependence of the hole concentration $p(T)[4,5]$. In this article, we report on our investigation of the influence of electron irradiation on $p(T)$ in p-type $4 \mathrm{H}-\mathrm{SiC}$ epilayers by means of free carrier concentration spectroscopy (FCCS) [5,12-14].

\section{Experimental}

$10 \mu \mathrm{m}$-thick Al-doped 4H-SiC epilayers (Al-doping density: $\sim 5 \times 10^{15} \mathrm{~cm}^{-3}$ ) on n-type 4H-SiC (thickness: $375.9 \mu \mathrm{m}$, resistivity: $0.02 \Omega \mathrm{cm}$ ) were cut to a $1 \times 1 \mathrm{~cm}^{2}$ size. Ohmic metal (Al/Ti) was deposited on the four corners of the surface of the sample, and then the sample was annealed at

Author to whom correspondence whould be addressed; matsuura@isc.osakac.ac.jp 
$900{ }^{\circ} \mathrm{C}$ for $1 \mathrm{~min}$ in an Ar atmosphere to form good ohmic contact. The $p(T)$ was measured by the van der Pauw method in the temperature range from 135 to $580 \mathrm{~K}$ and in a magnetic field of $1.4 \mathrm{~T}$ using a modified MMR Technologies' Hall System. After this measurement, the sample was irradiated with 4.6 MeV electrons with $2.6 \times 10^{14} \mathrm{~cm}^{-2}$ fluence, and then the $p(T)$ for the sample was measured.

\section{Results and Discussion}

The open circles and diamonds in Fig. 1 represent the measured $p(T)$ for the unirradiated and irradiated samples. It is clear from the figure that the $p(T)$ is reduced by electron irradiation. The possible origins of this reduction in $p(T)$ by irradiation are as follows: 1) a decrease in the acceptor density because substitutional acceptors are moved into the interstitial sites or because the bonds between the substitutional acceptor and the nearest neighbor atom are broken, or 2) the creation of hole traps or donor-like defects, which capture holes emitted from the acceptors.

In order to investigate acceptor densities and hole-trap density with $p(T)$, FCCS is used. The FCCS is a graphical peak analysis method for determining the densities and energy levels of acceptor species and hole traps using $p(T)$ without any assumptions regarding acceptor species and hole traps. Using an experimental $p(T)$, the FCCS signal is defined as $[4,5,13,14]$

$$
H\left(T, E_{\text {ref }}\right) \equiv \frac{p(T)^{2}}{(k T)^{5 / 2}} \exp \left(\frac{E_{\text {ref }}}{k T}\right)
$$

The FCCS signal has a peak at the temperature corresponding to each acceptor level or hole-trap level, where $E_{\text {ref }}$ is the parameter that can shift the peak temperature of the FCCS signal within the temperature range of the measurement, and $k$ is the Boltzmann constant. From each peak, the density and energy level of the corresponding acceptor or hole trap can be determined accurately.

On the other hand, $H\left(T, E_{\text {ref }}\right)$ is theoretically expressed as $[13,14]$

$$
H\left(T, E_{\text {ref }}\right)=\sum_{i} \frac{N_{\mathrm{A} i}}{k T} \exp \left(-\frac{\Delta E_{\mathrm{A} i}-E_{\mathrm{ref}}}{k T}\right) I\left(\Delta E_{\mathrm{A} i}\right)-\frac{N_{\text {comp }} N_{\mathrm{V} 0}}{k T} \exp \left(\frac{E_{\mathrm{ref}}-\Delta E_{\mathrm{F}}}{k T}\right)
$$

and

$$
I\left(\Delta E_{\mathrm{A} i}\right)=\frac{N_{\mathrm{V} 0}}{g_{\mathrm{A}}+\exp \left(\frac{\Delta E_{\mathrm{F}}-\Delta E_{\mathrm{A} i}}{k T}\right)},
$$
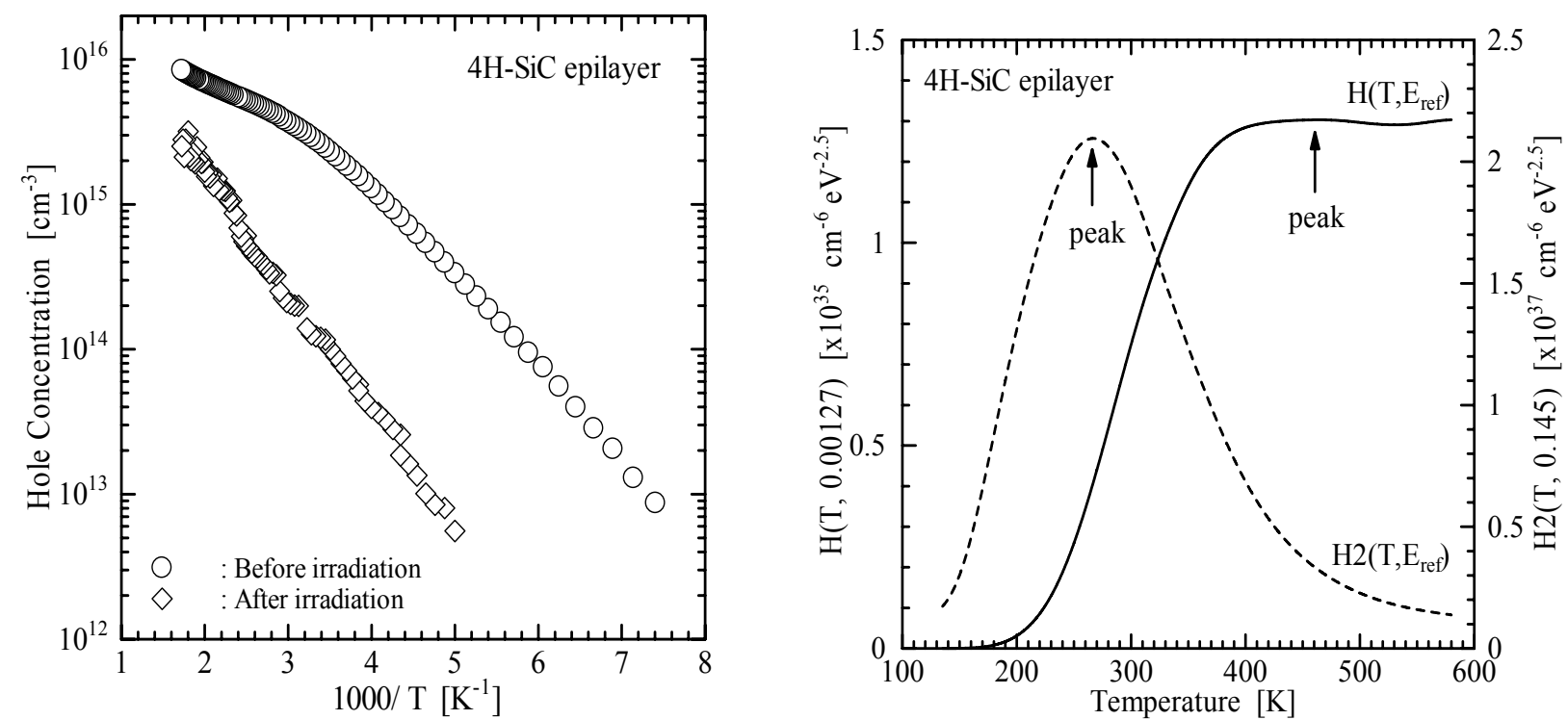

Fig. 1 Temperature dependent hole concentration. Fig. 2 FCCS signals. 
where $N_{\mathrm{A} i}$ and $\Delta E_{\mathrm{A} i}$ are the density and energy level of the $i$ th acceptor or hole trap, $N_{\text {comp }}$ is the compensating density, $\Delta E_{\mathrm{F}}$ is the Fermi level, $g_{\mathrm{A}}$ is the degeneracy factor for acceptors or hole traps, $N_{\mathrm{v} 0}=2\left(2 \pi m_{\mathrm{h}}^{*} / h^{2}\right)^{3 / 2}, m_{\mathrm{h}}^{*}$ is the hole effective mass, and $h$ is Planck's constant. Here, all energy levels are measured from the valence band maximum.

The solid line in Fig. 2 represents the FCCS signal with $E_{\text {ref }}=1.27 \times 10^{-3} \mathrm{eV}$ for the unirradiated sample. Here, the FCCS signal is calculated by interpolating $p(T)$ with a cubic smoothing natural spline function at intervals of $0.1 \mathrm{~K}$. From the peak at $464.2 \mathrm{~K}$, the corresponding density $\left(N_{\mathrm{A} 2}\right)$ and energy level $\left(\Delta E_{\mathrm{A} 2}\right)$ are determined as $4.2 \times 10^{15} \mathrm{~cm}^{-3}$ and $365 \mathrm{meV}$.

The FCCS signal of $H 2\left(T, E_{\text {ref }}\right)$, in which the influence of $\Delta E_{\mathrm{A} 2}$ is removed [14], is calculated using

$$
H 2\left(T, E_{\mathrm{ref}}\right)=\frac{p(T)^{2}}{(k T)^{5 / 2}} \exp \left(\frac{E_{\mathrm{ref}}}{k T}\right)-\frac{N_{\mathrm{A} 2}}{k T} \exp \left(-\frac{\Delta E_{\mathrm{A} 2}-E_{\mathrm{ref}}}{k T}\right) I\left(\Delta E_{\mathrm{A} 2}\right),
$$

and is denoted by the broken line in Fig. 2. Here, $E_{\text {ref }}=0.145 \mathrm{eV}$. From this peak at $266.7 \mathrm{~K}$, the corresponding density $\left(N_{\mathrm{A} 1}\right)$ and energy level $\left(\Delta E_{\mathrm{A} 1}\right)$, and $N_{\text {comp }}$ are determined as $6.2 \times 10^{15} \mathrm{~cm}^{-3}$, $203 \mathrm{meV}$ and $3.4 \times 10^{13} \mathrm{~cm}^{-3}$, respectively.

In the same way as illustrated for the unirradiated sample, the densities and energy levels for the irradiated sample are determined and listed in Table 1. The value of $N_{\mathrm{A} 1}$ is clearly reduced by electron irradiation, while $N_{\mathrm{A} 2}$ appears unchanged.

To verify the values obtained by FCCS, the $p(T)$ is simulated with the values shown in Table 1 . The open circles and diamonds in Fig. 3 represent the experimental $p(T)$ for the unirradiated and irradiated samples, and the solid and broke lines represent the simulated $p(T)$ for the unirradiated and irradiated samples. Each line is in good agreement with the corresponding experimental $p(T)$, indicating that the values determined by FCCS are reliable.

From photoluminescence measurements [15] and Hall-effect measurements [16], the energy level of $\sim 200 \mathrm{meV}$ is ascribed to $\mathrm{Al}$ acceptors in $4 \mathrm{H}-\mathrm{SiC}$. Moreover, $N_{\mathrm{A} 1}$ is close to the Al-doping density, suggesting that the origin of $\Delta E_{\mathrm{A} 1}$ is the $\mathrm{A} 1$ acceptor. Although the possible origin of $\Delta E_{\mathrm{A} 2}$ is $\mathrm{B}$ with which $4 \mathrm{H}-\mathrm{SiC}$ is sometimes contaminated, on the other hand, the concentration of $\mathrm{B}$ in this epilayer, which was determined by secondary ion mass spectroscopy, was $<4 \times 10^{14} \mathrm{~cm}^{-3}$, and $\Delta E_{\mathrm{A}}$ of $\mathrm{B}$ was reported to be $285 \mathrm{meV}$ [16]. Therefore, the origin of $\Delta E_{\mathrm{A} 2}$ is unfortunately unknown.

The density of substitutional Al atoms, which act as acceptors, is found to be reduced by $4.6-\mathrm{MeV}$ electron irradiation. This is why the $p(T)$ is decreased by the irradiation. Similar phenomena have been reported in p-type $\mathrm{Si}$ : the $p(T)$ in

Table 1 Results obtained by FCCS.

\begin{tabular}{|l|c|c|}
\hline & $\begin{array}{l}\text { Before } \\
\text { irradiation }\end{array}$ & After irradiation \\
\hline$\Delta E_{\mathrm{A} 1}[\mathrm{meV}]$ & 203 & 206 \\
\hline$N_{\mathrm{A} 1}\left[\mathrm{~cm}^{-3}\right]$ & $6.2 \times 10^{15}$ & $8.2 \times 10^{14}$ \\
\hline$\Delta E_{\mathrm{A} 2}[\mathrm{meV}]^{-3}$ & 365 & 383 \\
\hline$N_{\mathrm{A} 2}\left[\mathrm{~cm}^{-3}\right]$ & $4.2 \times 10^{15}$ & $3.4 \times 10^{15}$ \\
\hline$N_{\text {comp }}\left[\mathrm{cm}^{-3}\right]$ & $3.4 \times 10^{13}$ & $7.4 \times 10^{14}$ \\
\hline
\end{tabular}

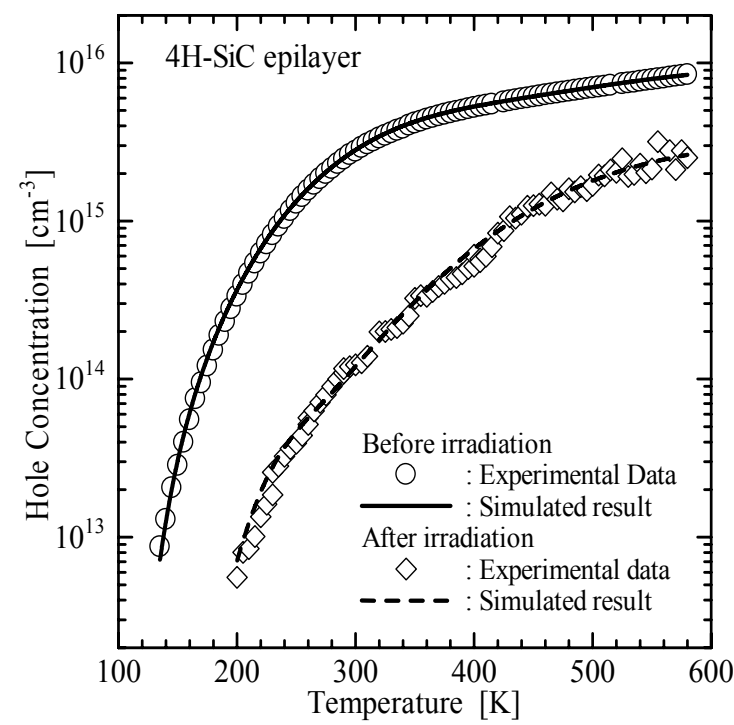

Fig. 3 Comparison between experimental and simulated $p(T)$. 
B-doped $\mathrm{Si}$ was reduced by $10-\mathrm{MeV}$ proton irradiation because of the decrease in the $\mathrm{B}$ acceptor density in $\mathrm{Si}$ [5].

The decrease in the Al acceptor density by irradiation is assumed to arise from the movement of the substitutional Al atoms into the interstitial sites, or from the bond-breaking between the substitutional $\mathrm{Al}$ atom and the nearest neighbor atom. Further research in this area is in progress.

\section{Summary}

The effect of electron irradiation on Al-doped 4H-SiC epilayers was investigated with Hall-effect measurements. The hole concentration in the p-type $4 \mathrm{H}-\mathrm{SiC}$ epilayer was reduced by $4.6-\mathrm{MeV}$ electron irradiation, and using FCCS the $200-\mathrm{meV}$ Al-acceptor density was found to be clearly decreased by the irradiation. On the other hand, the unknown $\sim 370-\mathrm{meV}$ defect density was unchanged. Therefore, the main reduction in $p(T)$ by irradiation resulted from the decrease of $\mathrm{Al}$ acceptor, not from the creation of hole traps or donor-like defects.

\section{References}

[1] A.L. Barry, B. Lehman, D. Fritsch and D. Bräuning: IEEE Trans. Nucl. Sci. Vol. 38 (1991), p. 1111.

[2] S.M. Sze: Physics of Semiconductor Devices (Wiley, New York, 1981) $2^{\text {nd }}$.

[3] T. Hisamatsu, O. Kawasaki, S. Masuda, T. Nakao and Y. Wakow: Solar Energy Mater. Solar Cells Vol. 50 (1998), p. 331.

[4] H. Matsuura, Y. Uchida, T. Hisamatsu and S. Matsuda: Jpn. J. Appl. Phys. Vol. 37 (1998), p. 6034.

[5] H. Matsuura, Y. Uchida, N. Nagai, T. Hisamatsu, T. Aburaya and S. Matsuda: Appl. Phys. Lett. Vol. 76 (2000), p. 2092.

[6] H.J. von Bardeleben and J.L. Cantin: Mater. Sci. Forum Vol. 353-356 (2001), p. 513.

[7] H.J. von Bardeleben, J.L. Cantin, P. Baranov and E.N. Mokhov: Mater. Sci. Forum Vol. 353-356 (2001), p. 509.

[8] Th. Lingner, S. Greulich-Weber and J.-M. Speath: Mater. Sci. Forum Vol. 353-356 (2001), p. 505.

[9] I.V. Ilyin, E.N. Mokhov and P.G. Baranov: Mater. Sci. Forum Vol. 353-356 (2001), p. 521.

[10] V.Ya. Bratus, I.N. Makeeva, S.M. Okulov, T.L. Petrenko, T.T. Petrenko and H.J. von Bardeleben: Mater. Sci. Forum Vol. 353-356 (2001), p. 517.

[11] A.A. Rempel, K. Blaurock, K.J. Reichle, W. Sprengel and H.-E. Schaefer: Mater. Sci. Forum Vol. 389-393 (2002), p. 485.

[12] H. Matsuura and K. Sonoi: Jpn. J. Appl. Phys. Vol. 35 (1996), p. L555.

[13] H. Matsuura, Y. Masuda, Yi Chen and S. Nishino: Jpn. J. Appl. Phys. Vol. 39 (2000), p. 5069.

[14] H. Matsuura, K. Morita, K. Nishikawa, T. Mizukoshi, M. Segawa and W Susaki: Jpn. J. Appl. Phys. Vol. 41 (2002), p. 496.

[15] M. Ikeda, H. Matsunami and T. Tanaka: Phys. Rev. B Vol. 22 (1980), p. 2842.

[16] T. Troffer, M. Schadt, T. Frank, H. Itho, G. Pensl, J. Heindl, H.P. Strunk and M. Maier: Phys. Status Solidi A Vol. 162 (1997), p. 277. 